\title{
Guest editorial: immunotherapy for hematological malignancies: the quest to overcome tolerogenic drive
}

\author{
Norimitsu Kadowaki
}

Received: 9 December 2013/Revised: 24 December 2013/Accepted: 24 December 2013/Published online: 7 January 2014

(C) The Japanese Society of Hematology 2014

How easily a healthy body can defend itself against ordinary pathogens! If only it was possible to do the same against self-derived malignancies. Unfortunately, however, the physiology of the immune system discourages such a persistent fight by establishing the overwhelming hurdle of "tolerance". For this reason, tumor immunotherapy stands as an ultimate challenge to the fundamental basis of immunology. Will we ever win this battle? Recent remarkable advances in related fields are finally beginning to make tumor immunologists confident enough to answer in the affirmative.

In fact, it has been hematologists who have led the field of tumor immunotherapy through their work in allogeneic hematopoietic stem cell transplantation, which exploits the power of graft-versus-tumor effects. However, this is inevitably accompanied by graft-versus-host disease, which precludes elderly patients, who represent a major population suffering from hematological malignancies, from receiving allogeneic transplantation. Clearly, we must develop novel therapies for such patients.

In contrast to the tremendous power of allogeneic reaction, autologous antitumor responses are usually feeble. Heightening them to a clinically meaningful level is a daunting task in the face of the driving force of tolerance. However, rational strategies for overcoming each step in the series of tolerogenic mechanisms have gradually brought immunotherapy into the arena of cancer therapy.

Positive and negative components that, respectively, enhance and suppress immune responses are present in the immune system, as in other physiological systems, and are

N. Kadowaki $(\bowtie)$

Department of Hematology and Oncology, Graduate School of

Medicine, Kyoto University, Kyoto, Japan

e-mail: kadowaki@kuhp.kyoto-u.ac.jp responsible for maintaining homeostasis. The primordial positive component that triggers virtually any type of immune response is innate immunity. The main reason why the immune system efficiently combats pathogens is that innate immune cells express an abundant array of receptors that recognize various molecules found in microbes. Such recognition provokes intense inflammatory responses and the activation of dendritic cells (DCs), the most potent antigen-presenting cells for $\mathrm{T}$ cells. Inflammation and activated DCs subsequently initiate effective antigen-specific immune responses, that is, adaptive immunity. This "innate immunity-DC-adaptive immunity" axis is the essence of the positive immune component.

To prevent immune responses to autologous or innocuous antigens, and to avoid excessive responses to noxious antigens, the immune system has several layers of negative (suppressive) components. First of these is the induction of central tolerance in the thymus, through which high-affinity self-reactive $\mathrm{T}$ cells are eliminated. To assure tolerance to innocuous antigens in the periphery, peripheral tolerance is maintained by several mechanisms, among which regulatory $\mathrm{T}$ cells play a key role. Furthermore, negative signals transmitted to $\mathrm{T}$ cells through inhibitory receptors, such as cytotoxic T-lymphocyte-associated antigen-4 (CTLA-4) and programmed cell death protein-1 (PD-1), represent crucial molecular mechanisms for the timely termination of $\mathrm{T}$ cell responses [1]. In addition, tumor tissues co-opt certain immunosuppressive components, including regulatory T cells, PD-1 ligands, and myeloid-derived suppressor cells, thus creating a microenvironment hostile to tumorreactive $\mathrm{T}$ cells.

These positive and negative components provide targets for provoking antitumor immune responses. In this issue, Dr. Bocchia reviews peptide vaccines derived from antigens that are preferentially expressed by tumor cells, thus 
circumventing self-tolerance at least in part. In addition, developing appropriate adjuvants that are combined with peptide vaccines will be important for improving efficacy by triggering innate immune responses [2]. Dr. Kitawaki reviews DC vaccines that exploit the power of the most potent $T$ cell stimulator. Dr. Fujiwara and Dr. Turtle review adoptive immunotherapy using $\mathrm{T}$ cells modified with T-cell receptor or chimeric antigen receptor genes, which bypasses tolerance induced in vivo in cancer patients by transferring tumor-reactive $\mathrm{T}$ cells cultured ex vivo. Theoretically, all of these methods can be combined with blockade of the negative immune components to enhance efficacy. In particular, blocking CTLA-4 and/or PD-1 signals, which have been shown to induce remarkable clinical effects by themselves [3], are immediate candidates. The anti-CCR4 monoclonal antibody, which eliminates regulatory $\mathrm{T}$ cells, also represents a promising enhancer of antitumor immunity. Such blockade of "immune checkpoints" will constitute an integral component of tumor immunotherapy [1].

Although immunotherapy is generally considered to be "safe", we need to be careful about side effects as efficacy increases. For example, targeting antigens that are expressed by normal cells can cause severe adverse events [4]. Also, blocking inhibitory pathways hardwired into the immune system, which are crucial for maintaining selftolerance and modulating the amplitude of physiological immune responses, cause collateral tissue damage [1]. We need to take care to minimize these side effects to an acceptable level.

In any event, tumor immunotherapy has certainly begun to come of age. We can expect to observe remarkable advances in the translation of basic findings to clinical applications. In the field of hematological malignancies, immunotherapy will be applied mainly to elderly patients ineligible for allogeneic transplantation. Furthermore, if "autologous" immunotherapy turns out to be truly effective, it may replace part of "allogeneic" immunotherapy as we pursue safer cancer therapies.

\section{References}

1. Pardoll DM. The blockade of immune checkpoints in cancer immunotherapy. Nat Rev Cancer. 2012;12:252-64.

2. Coffman RL, Sher A, Seder RA. Vaccine adjuvants: putting innate immunity to work. Immunity. 2010;33:492-503.

3. Wolchok JD, Kluger H, Callahan MK, Postow MA, Rizvi NA, Lesokhin AM, et al. Nivolumab plus ipilimumab in advanced melanoma. N Engl J Med. 2013;369:122-33.

4. Morgan RA, Yang JC, Kitano M, Dudley ME, Laurencot CM, Rosenberg SA. Case report of a serious adverse event following the administration of $\mathrm{T}$ cells transduced with a chimeric antigen receptor recognizing ERBB2. Mol Ther. 2010;18:843-51. 